\title{
Biomechanical bearing-based typing method for osteonecrosis of the femoral head: ABC typing
}

\author{
ZHENNAN ZHANG, TONG YU, LIMIN XIE, YUBIN LI, XUN KE, \\ YANG LIU, SONGMIN HUANG, HAO DENG and YANG BAI
}

Department of Orthopedics, Guanganmen Hospital, China Academy of Chinese Medical Sciences, Beijing 100053, P.R. China

Received December 17, 2017; Accepted June 1, 2018

DOI: $10.3892 /$ etm.2018.6488

\begin{abstract}
Type classification of osteonecrosis of the femoral head $(\mathrm{ONFH})$ is important for collapse prediction in $\mathrm{ONFH}$, which depends on a complexity of factors. At present, most typing is based on single factors, including the location or size of the necrosis, or the bone repair capacity after ONFH, and is therefore limited. The present study proposes an 'ABC' method for ONFH typing based on biomechanics and the stress distribution characteristics of the femoral head's bone trabeculae. In total, $132 \mathrm{ONFH}$ patients (223 hips) were enrolled at Guanganmen Hospital (Beijing, China). Each of the hip joints included was subjected to computerized tomography and/or magnetic resonance imaging. The images with the maximum necrotic area in the coronal femoral head were selected, and the femoral head's maximum transverse diameter was divided into three pillars (A, B and C, from the outside to the inside) according to a 3:4:3 diameter ratio. ONFH was typed according to the number of pillars involved in the necrosis. Differences in the collapse rate of different ONFH types, and the correlation between the theoretical collapse risk and the observed collapse rate was analysed. The ONFH types significantly differed in their collapse rate $\left(\chi^{2}=76.93, \mathrm{P}<0.001\right)$ in the following order: $\mathrm{A}-\mathrm{C}(88.6 \%)>\mathrm{AB}(74.1 \%)>\mathrm{BC}(52.4 \%)>\mathrm{A}(50 \%)>\mathrm{B}(9.5 \%)>\mathrm{C}$ $(0 \%)$. The collapse risk was significantly correlated with the collapse rate (correlation coefficient $\mathrm{R}=1$ ). The types $\mathrm{A}-\mathrm{C}$ and $\mathrm{AB}$ had high collapse rates/risks, whereas types $\mathrm{B}$ and $\mathrm{C}$ had a satisfactory prognosis. The ABC typing proposed in the present study is thus suitable for collapse risk prediction in
\end{abstract}

Correspondence to: Professor Limin Xie, Department of Orthopedics, Guanganmen Hospital, China Academy of Chinese Medical Sciences, 5 Beixiange Street, Xicheng, Beijing 100053, P.R. China

E-mail: drxlm@126.com

Abbreviations: ONFH, osteonecrosis of the femoral head; ARCO, association research circulation osseous; T1WI, T1-weighted imaging

Key words: femoral head, osteonecrosis, ABC typing, collapse, prediction
ONFH. Type classification using this method may provide a valuable reference for selecting regimens for ONFH treatment.

\section{Introduction}

Osteonecrosis of the femoral head (ONFH) is one of the most common challenging orthopaedic conditions and poses a great threat to human health $(1,2)$. ONFH has a high occurrence rate; in China, an estimated 5,000,000-7,500,000 ONFH patients currently require treatment and the number of novel cases per year is as high as 150,000-300,000, and thus, it ranks first among hip joint diseases $(3,4)$. ONFH frequently occurs in young and middle-aged populations. Without any timely and effective treatment, ONFH develops to femoral collapse in $\sim 80 \%$ of patients within 1-4 years, ultimately causing severe damage to the entire hip joint (5-9).

At present, ONFH patients without collapse or with only mild collapse are primarily subjected to joint preservation. However, once noticeable femoral collapse occurs, the efficacy of preservation treatment is greatly decreased. Consequently, patients must undergo total hip arthroplasty (THA) $(8,10,11)$. Furthermore, artificial prostheses have a limited service life. For this reason, most young patients receive repeated THA, which is not only painful for them but also greatly increases the economic burden on their family and society (12). Therefore, whether collapse occurs after ONFH is critically relevant to the prognosis of ONFH and the guidance of clinical treatment, and accordingly, the prediction of collapse after ONFH has long been a clinical research focus.

Numerous studies have indicated that whether femoral collapse occurs after ONFH is associated with the location and size of the necrosis, as well as with the bone remodelling ability, and most of the studies that evaluated the Association Research Circulation Osseous (ARCO) stage, Japanese Investigation Committee (JIC) type, the sum of the necrotic angles, the proportion of the necrotic area and the proportion of the proximal sclerotic rim have indicated that a large necrotic area, necrosis located at the lateral part of the femoral head and poor bone remodelling ability after necrosis are risk factors for the collapse of the osteonecrosis-affected femoral head (13-20). However, due to the complexity of the issue itself and the limitations of imaging examination, all of the above studies predicted the occurrence of the collapse from only one perspective. The ARCO (21) and Steinberg 
systems (22) have been widely applied in ONFH staging. These systems propose subtypes of ONFH based on its severity and its characteristics at different stages. However, their classifications of different subtypes of ONFH primarily focus on the necrotic area based on imaging features of ONFH at different stages, without taking the necrotic location into account. Li et al (23) of the China-Japan Friendship Hospital (CJFH) proposed a novel typing method based on the necrotic location and the necrotic area. They postulated that ONFH may be classified into the medial (M), central (C) and lateral (L) types, and the L type may be further classified into L1, L2 and L3; the M and C types have a good prognosis, followed by the L1 type, while the L2 and L3 types have the poorest prognosis. This typing method may be used for direct demarcation of the necrotic area and is not influenced by any acetabular anatomical factors. The method results in smaller errors in predicting collapse compared with other methods and is therefore more satisfactory than other methods in clinical practice. However, the CJFH method has its own limitations. First, it utilizes the medial slice of the coronal section based on computerized tomography (CT) or magnetic resonance imaging (MRI). It is generally thought that the medial slice of the femoral head is the site that bears the maximal stress. However, the maximal necrotic area may better reflect the actual ONFH status, as it is the mechanically weak area where collapse tends to occur. Therefore, the maximal necrotic area may provide better information for accurate ONFH prognosis prediction. In most patients with $\mathrm{ONFH}$, the maximal plane of the necrotic focus is not located at the medial plane, but instead slightly anterior or posterior (24). Furthermore, the $\mathrm{CJFH}$ method fails to clarify the mechanical foundation and detailed demarcation of the three-pillar structure. This drawback causes a degree of randomness in the demarcation of the femoral three-pillar structure in clinical practice, thereby increasing the uncertainty of the prediction results.

The structure of bones appears to be adapted to performing specific functions. While the bone structure determines what functions the bone is able to perform, the functions also influence changes in the bone structure, i.e., a change in either structure or function causes a change in the other to maintain the balance between them (25-27). According to Wolff's law, bone trabeculae are not arranged out of order, but rather along the direction of the primary compression to present an optimal stress-bearing characteristic (28). According to this system, the proximal trabeculae of the femoral head is divided into primary compression trabeculae, secondary compression trabeculae, primary tensile trabeculae, secondary tensile trabeculae, and pertrochanteric trabeculae, and the inside of the femoral head comprises the primary compression trabeculae and primary tensile trabeculae. A stress test on the proximal femoral head demonstrated that the stress on the primary tensile trabeculae was noticeably reduced under a normal physiological load, whereas the primary compression trabeculae became the primary stress region (29), suggesting that the primary compression trabeculae serve as the primary weight-bearing structure under a normal physiological load and as the primary mechanical support site, and that the distribution limits of the compression trabeculae may be regarded as a basis for three-pillar demarcation. Analysis of the characteristics of the collapse site in ONFH indicated that the anterolateral part of the proximal femoral head is the most common site of collapse. This part of the proximal femoral head contains sparsely distributed primary compression trabeculae, suggesting an important weight-bearing role for the compression trabeculae in the femoral head (30). The application of the three-pillar theory was first proposed by Herring et al (31) in 1992 for the typing of childhood ONFH (i.e., Legg-Calvé-Perthes' disease). According to this theory, the integrity of the lateral pillar provides an effective biomechanical shielding effect for the central part of the femoral head, preventing collapse. If the lateral pillar is structurally impaired as a result of disease, its shielding effect disappears and collapse of the femoral head becomes unavoidable.

In the light of these theories, a previous study by our group assessed frontal X-ray images of the bilateral hips of healthy volunteers (32). The lateral, central and medial pillars were identified from the intersection points of the lateral and medial rims of the primary compression trabeculae with the maximum transverse diameter of the femoral head, and the width ratios of the lateral, central and medial pillars of the femoral head were then calculated to set a standard of demarcating the three pillars. The results indicated that the ratio of the three pillars of the femoral head in terms of biomechanical weight bearing was $\sim 3: 4: 3$ (32). Based on this result and on the plane with the maximal ONFH area, the present study proposes a novel typing method for ONFH, referred to as ABC typing.

\section{Materials and methods}

General data. A total of 132 patients (223 hips) with ONFH who received treatment at Guanganmen Hospital (Beijing, China) from October 2012 to April 2015 were enrolled in the present study. The diagnostic criteria were based on the Chinese Experts' Consensus on the Diagnosis and Treatment of Osteonecrosis of the Femoral Head in Adults from 2012 (1). Among the patients, 94 were males and 38 were females. Their age ranged from 18 to 78 years, with a median age of 43 years. In total, 41 patients had ONFH in one hip and 91 in the bilateral hips, and the median course of the disease was 50 months, ranging from 36 to 147 months. Within the cohort, 45 cases (34.1\%) had alcohol-induced ONFH; 57 cases (43.2\%) had steroid-induced ONFH and 30 cases $(22.7 \%)$ had idiopathic ONFH. According to the ARCO staging criteria for ONFH (21), 5 hips were stage I; 79 were stage II, 106 were stage III and 33 were stage IV.

The inclusion criteria for the present study were as follows: i) Diagnosis of ONFH according to the abovementioned criteria (1); ii) an ARCO stage of $\geq \mathrm{I}$; iii) patient age of $\geq 18$ years; iv) MRI or CT of both hip joints available (for patients with an ARCO stage of $\geq \mathrm{II}$ ); v) a natural disease course of $\geq 3$ years if no collapse of the femoral head had occurred; and vi) informed consent provided by the patient. Patients were excluded from the present study if they met any of the following criteria: i) Traumatic ONFH or ONFH complicated by other joint diseases (including bone neoplasms, rheumatoid arthritis, ankylosing spondylitis, joint tuberculosis or suppurative arthritis); ii) a severe congenital anomaly in the hip joint; iii) a history of hip joint surgery; and iv) mental disorders. 
Grouping. An ARCO stage of $\geq$ III was defined as the collapse of the osteonecrosis-affected femoral. The patients were divided into a collapse and a non-collapse group.

CT scanning. A Siemens 64-slice dual-source spiral CT (Siemens AG, Munich, Germany) was used for successive scanning of the bilateral hip joints along the cross-section. The scanning parameters were as follows: Voltage, $120 \mathrm{kV}$; electric current, $60 \mathrm{~mA}$; and slice thickness for bone tissue window scanning, $0.75 \mathrm{~mm}$. The images obtained were exported in bmp format.

MRI. A GE-signal $1.5 \mathrm{~T}$ superconducting nuclear magnetic resonance analyser (GE Healthcare, Little Chalfont, UK) was utilized to collect T1-weighted images (T1WI) of the coronal section of the femoral head. The scanning parameters for T1WI were as follows: Echo time, $12 \mathrm{msec}$; repetition time, 560 msec; coil, USC_S12; number of excitations, 2.00; matrix, $320 \times 256$; field of view, 24; slice thickness, $3.0 \mathrm{~mm}$; and slice interval, $1 \mathrm{~mm}$.

$A B C$ typing method. Based on the three pillars defined by Herring in Legg-Calvé-Perthes disease (31) and the measurements and observations of the mechanical distribution characteristics of bone trabeculae in X-ray images, the coronal plane of the femoral head was divided into the lateral, central and medial pillars, which represented 30,40 and $30 \%$ of the total width of the femoral head, respectively. The detailed demarcation method was as follows: i) The slice with the maximal necrotic area was selected from the CT (or MRI T1WI) images for measurement; ii) The selected image was opened in Computer Aided Design software (AutoCAD 2012; Autodesk, San Francisco, CA, USA); iii) The femoral head was regarded as a circle to determine its centre, 'O'. A horizontal line was drawn across ' $\mathrm{O}$ ' and the circle was intersected at the points 'a' and 'd'. The line section 'ad' was the maximum transverse diameter of the femoral head; iv) The vertical lines L1 and L2 were drawn rectangular to the line 'ad', with the intersection points named ' $b$ ' and 'c', respectively, dividing 'ad' into 'ab', 'bc' and 'cd' with a length ratio of 3:4:3. The three sections divided by L1 and L2 corresponded to the three pillars A, B and C (Fig. 1).

According to the involvement of the three pillars in the necrotic focus, ONFH was classified into 6 types as follows (Fig. 2): Type A (lateral)-the lateral pillar was involved, but the central and medial pillars were intact; Type B (central)-the central pillar was involved, but the lateral and medial pillars were intact; Type $\mathrm{C}$ (medial)-the medial pillar was involved, but the central and lateral pillars were intact; Type AB (lateral double-pillar)-the lateral and central pillars were involved, but the medial pillar was intact; Type BC (medial double-pillar)-the central and medial pillars were involved, but the lateral pillar was intact; Type A-C (three-pillar)-all pillars were involved.

Collapse risk value assignment. The necrotic area is known to be positively associated with the risk of collapse $(14,17,18,20)$; therefore, the involvement of one, two or three pillars was scored as 1, 2 or 3 points, respectively. Furthermore, the collapse risk is high when the lateral pillar is involved (23); thus, the involvement of the medial, central or lateral pillar

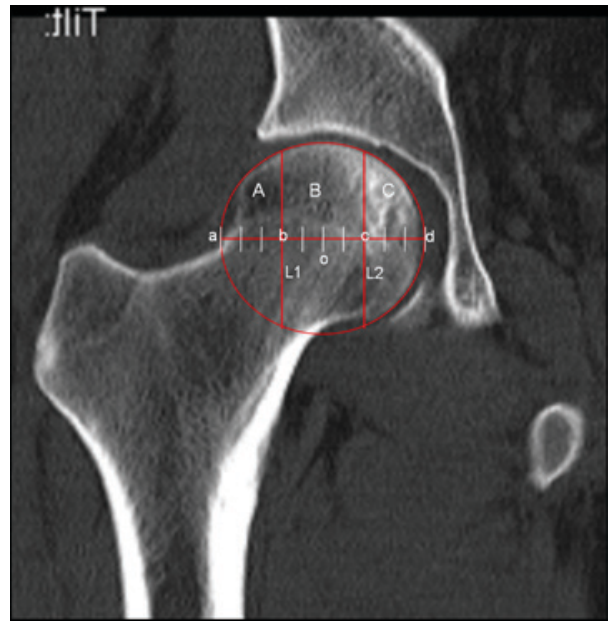

Figure 1. The ABC typing method proposed in the current study. Vertical lines for the maximum transverse diameters L1 and L2 were drawn. The lines divided the maximum transverse diameter with a length ratio of $3: 4: 3$; ' $a$ ' and ' $d$ ' are the intersection points of the horizontal line across the centre 'o' of the circle, and ' $b$ ' and 'c' are the intersection points of L1 and L2 with the horizontal line. The femoral head was divided into the A, B and C pillars (i.e., the lateral, central and medial pillars, respectively).



Figure 2. Mirror images of $\mathrm{ABC}$ typing. The necrotic area is indicated in pink. The upper panels show different ONFH types involving one pillar (A, B and C from left to right). The lower panels show different ONFH types involving more than one pillar ( $\mathrm{AB}, \mathrm{BC}$ and $\mathrm{AC}$ from left to right). $\mathrm{ONFH}$, osteonecrosis of the femoral head.

was assigned 1, 2 or 3 points, respectively. Based on these methods, the collapse risk was scored for different $\mathrm{ABC}$ types.

The differences in the occurrence of collapse in the different $\mathrm{ABC}$ types of ONFH were examined, and the correlation of the collapse risk value with the collapse rate was analysed. The image review, evaluation and scoring were performed by experienced orthopaedists.

Statistical analysis. Data were processed using SPSS 12.0 software (SPSS, Inc., Chicago, IL, USA). The differences in the collapse rate among different $\mathrm{ABC}$ types were tested using 
the chi-square method. The correlation between the collapse risk value and the collapse rate was analysed by calculating Spearman's rank correlation coefficient. All statistical tests were two-sided. $\mathrm{P}<0.05$ was considered to indicate a statistically significant difference.

\section{Results}

Baseline data. The collapse group included 166 hips and the non-collapse group included 57 hips. In the collapse group, 72 patients were males and 24 were females, and in the non-collapse group, 22 were males and 14 were females. No significant difference was observed in the gender ratio between the groups $\left(\chi^{2}=1.38, \mathrm{P}=0.24\right)$. The average age of the collapse group was $44 \pm 3$ years and that of the non-collapse group was $45 \pm 4$ years. No significant difference in age was observed between the two groups $(\mathrm{t}=-0.93, \mathrm{P}=0.35)$. Finally, the average disease course of the collapse group was $4.3 \pm 1.2$ years, and that of the non-collapse group was $4.0 \pm 0.7$ years $(t=0.98$, $\mathrm{P}=0.33$ ).

Distribution of the ONFH types. The proportions of the A, B, $\mathrm{C}, \mathrm{AB}, \mathrm{BC}$ and $\mathrm{A}-\mathrm{C}$ types among the total enrolled cases were $0.9,9.4,1.3,12.1,9.4$ and $66.8 \%$, respectively. These results suggest that ONFH involving only the lateral or the medial pillar was rare in clinical practice, whereas the involvement of all three pillars was common (Table I).

ABC typing of the collapse and non-collapse groups. Among the 166 hips in the collapse group, one $(0.6 \%)$ was type A, two $(1.2 \%)$ were type B, $20(12.1 \%)$ were type AB, 11 (6.6\%) were type BC and $132(79.5 \%)$ were type A-C. Among the 57 hips in the non-collapse group, one (1.9\%) was type A, $19(33.3 \%)$ were type $\mathrm{B}$, three $(5.3 \%)$ were type $\mathrm{C}$, seven $(12.3 \%)$ were type AB, 10 (17.5\%) were type BC and 17 (29.8\%) were type A-C (Table II). Significant differences were observed between the two groups regarding all types $(\mathrm{P}<0.001)$.

Collapse occurrence in the different types of ONFH. Among the 223 hips with ONFH, collapse occurred in 50, 9.5, 0.0, $74.1,52.4$ and $88.6 \%$ of hips of the type A, B, C, AB, BC and A-C, respectively $\left(\chi^{2}=76.93, \mathrm{P}<0.001\right)$. For the different $\mathrm{ONFH}$ types, collapse occurred most frequently in the following order: $\mathrm{A}-\mathrm{C}>\mathrm{AB}>\mathrm{BC}>\mathrm{A}>\mathrm{B}>\mathrm{C}$ (Table III).

Pairwise comparison of the collapse occurrence between the different types of ONFH revealed significant differences (Table III). However, as the sample size of certain groups was small (for instance, there were only 2 cases of type A and 3 cases of type $\mathrm{C}$ ), the statistical power may have been low. Therefore, the data were re-analysed regarding the extent and location of ONFH using a lower number of categories. Based on the number of pillars involved (i.e., the necrotic area), the joints were stratified into one-, two- and three-pillar involvement groups. In addition, according to whether the necrosis affected the lateral pillar, the joints were stratified into lateral pillar-involving and non-lateral pillar-involving groups. The analysis of the merged data revealed the following: The groups with different numbers of pillars involved exhibited significant differences $\left(\chi^{2}=72.20, \mathrm{P}<0.001\right)$, in the following order: The three-pillar group $(88.6 \%)>$ the two-pillar group $(64.6 \%)>$ the
Table I. ABC typing of the hips with osteonecrosis of the femoral head $(n=223)$.

\begin{tabular}{lc}
\hline ABC type & $\mathrm{n}(\%)$ \\
\hline A & $2(0.9)$ \\
B & $21(9.4)$ \\
C & $3(1.3)$ \\
AB & $27(12.1)$ \\
BC & $21(9.4)$ \\
A-C & $149(66.8)$
\end{tabular}

one-pillar group (11.5\%; Table IV). Furthermore, the lateral pillar-involving group and the non-lateral pillar-involving groups exhibited a significant difference in the collapse rate (86.0 vs. $28.9 \% ; \chi^{2}=61.47, \mathrm{P}<0.001$; Table V).

Correlation between the collapse risk and the collapse rate. The collapse risk values were assigned according to the ABC type (i.e., 6 categories) to verify the correlation between the collapse risk and the collapse rate. The collapse risk was noticeably correlated with the collapse rate $(\mathrm{R}=1$; Table VI).

\section{Discussion}

The CJFH typing method applied in the present study is based on the three-pillar theory, considers the necrotic location and the necrotic area (23), and its proposal has greatly increased the accuracy of collapse prediction (30). However, this typing method has certain limitations. First, in clinical practice, a relatively large proportion of cases suffer from ONFH located in somewhat anterior or posterior areas, therefore the medial plane is less likely to reflect the actual necrotic state compared with the maximal plane (33). Particularly, for patients with a small necrotic area, the medial plane may not even reveal the necrotic area (33). In a previous study by our group, the mechanical distribution of ONFH was analysed using the finite element method, indicating that the most noticeable stress concentration was at the border between the necrotic tissue and the normal tissue, where collapse was most likely to occur (34). Based on our experience, using the CT or MRI slice with the maximum ONFH area for typing has higher practical significance in the clinical judgement of the prognosis of ONFH. Furthermore, clinicians may have diverse opinions regarding which CT/MRI slice should be used as the image of the medial plane of the femoral head (35). As a consequence, selection errors may occur. By contrast, the slice with the maximum necrotic area is relatively definite on CT and on MRI, so it is easy to select and convenient to use, which may increase the accuracy and repeatability of the clinical application. Therefore, in the present study, the CT or MRI slice with the maximum coronal necrotic focus was used for $\mathrm{ABC}$ typing to predict the occurrence of collapse in $\mathrm{ONFH}$. In addition, CJFH typing fails to quantitate how much of the lateral pillar must be intact to prevent the occurrence of femoral collapse. Furthermore, CJFH typing does not cover 
Table II. Comparison of the proportion of hips with different ABC types of osteonecrosis of the femoral head in the collapse group $(n=166)$ and the non-collapse group $(n=57)$.

\begin{tabular}{lcccccr}
\hline Collapse & A & B & C & AB & BC & A-C \\
\hline Yes & $1(0.6)$ & $2(1.2)$ & $0(0.0)$ & $20(12.1)$ & $11(6.6)$ & $132(79.5)$ \\
No & $1(1.9)$ & $19(33.3)$ & $3(5.3)$ & $7(12.3)$ & $10(17.5)$ & $17(29.8)$ \\
\hline
\end{tabular}

Values are expressed as $\mathrm{n}(\%) \cdot \chi^{2}=76.93, \mathrm{P}<0.001$.

Table III. Collapse rates in the different types of osteonecrosis of the femoral head.

\begin{tabular}{lcc}
\hline ABC type & Hips $(\mathrm{n})$ & Collapses, $\mathrm{n}(\%)$ \\
\hline A & 2 & $1(50.0)$ \\
B & 21 & $2(9.5)$ \\
C & 3 & $0(0.0)$ \\
AB & 27 & $20(74.1)$ \\
BC & 21 & $11(52.4)$ \\
A-C & 149 & $132(88.6)$ \\
Total & 223 & $166(74.4)$ \\
\hline
\end{tabular}

$\chi^{2}=76.93, \mathrm{P}<0.001$ between all types (collectively as well as pairwise).

Table IV. Frequency of collapse of femoral heads with osteonecrosis stratified by the number of pillars involved.

\begin{tabular}{lcc}
\hline Pillars & Hips (n) & Collapsed femoral heads, $\mathrm{n}(\%)$ \\
\hline 1 & 26 & $3(11.5)$ \\
2 & 48 & $31(64.6)$ \\
3 & 149 & $132(88.6)$ \\
Total & 223 & $166(74.4)$ \\
\hline
\end{tabular}

$\chi^{2}=72.20, \mathrm{P}<0.001 .1$, one pillar involved; 2 , two pillars involved; and 3 , three pillars involved.

all ONFH conditions (e.g., when only the lateral pillar or the central pillar is affected by necrosis), which further limits its clinical application. Finally, although CJFH typing is based on the three-pillar theory, it fails to specify detailed demarcation criteria. In the present study, an ABC typing method was proposed based on the mechanical distribution characteristics of the bone trabeculae inside the femoral head, and a detailed approach that provides criteria for the demarcation of the three-pillar structure was applied.

The present study indicated that the proportions of types A, $\mathrm{B}$ and $\mathrm{C}$ in $\mathrm{ONFH}$ were $0.9,9.4$ and $1.3 \%$, respectively, whereas the proportion of type A-C was as high as $66.8 \%$; these results indicate that ONFH involving only a single pillar is a rare clinical phenomenon. By contrast, ONFH involving three pillars is the most frequent clinical condition, followed by the involvement of two pillars. The incidence of collapse
Table V. Proportion of collapsed hips in patients with osteonecrosis of the femoral head stratified based on involvement of the lateral pillar.

\begin{tabular}{lcc}
\hline $\begin{array}{l}\text { Lateral } \\
\text { pillar involvement }\end{array}$ & Hips (n) & $\begin{array}{c}\text { Collapsed femoral } \\
\text { heads, n (\%) }\end{array}$ \\
\hline Yes & 178 & $153(86.0)$ \\
No & 45 & $13(28.9)$ \\
Total & 223 & $166(74.4)$ \\
\hline
\end{tabular}

$\chi^{2}=61.47, \mathrm{P}<0.001$.

Table VI. Correlation between the risk of collapse and the collapse rate.

\begin{tabular}{lrccccc}
\hline Item & A & B & C & AB & BC & A-C \\
\hline Risk value (score) & 4 & 3 & 2 & 7 & 5 & 9 \\
Collapse rate (\%) & 50 & 9.5 & 0 & 74.1 & 52.4 & 88.6 \\
\hline
\end{tabular}

Correlation coefficient $\mathrm{R}=1$.

in types $\mathrm{A}, \mathrm{B}, \mathrm{C}, \mathrm{AB}, \mathrm{BC}$ and $\mathrm{A}-\mathrm{C}$ was $50,9.5,0,74.1,52.4$ and $88.6 \%$, respectively, with an order, from high to low, of $\mathrm{A}-\mathrm{C}>\mathrm{AB}>\mathrm{BC}>\mathrm{A}>\mathrm{B}>\mathrm{C}$, and the differences were significant. This result indicates that $\mathrm{ABC}$ typing is able to determine the probability of collapse in ONFH with different areas/extent of necrotic involvement, thereby offering value in clinical application. In addition, the present study indicated that the collapse rate of ONFH involving three pillars was $88.6 \%$, that of ONFH involving two pillars was $64.6 \%$ and that of ONFH involving one pillar was $11.5 \%$. ONFH involving the lateral pillar had a collapse rate of $86.0 \%$, which was significantly different from that for ONFH without lateral pillar involvement. These results suggest that the size of the necrotic area and whether the lateral pillar is involved are influential factors regarding the collapse of the osteonecrosis-affected femoral. In the present study, the collapse rate of type $\mathrm{C} \mathrm{ONFH} \mathrm{was} 0 \%$, and collapse occurred in only two of the 21 hips (9.5\%) with type B ONFH, which suggests that collapse does not tend to occur in ONFH with a small necrotic focus that involves only the medial or central pillar. By contrast, the collapse rates of types $\mathrm{A}-\mathrm{C}$ and $\mathrm{AB}$ were as high as 88.6 and $74.1 \%$, respectively, which suggests that collapse is likely to occur in ONFH with a 
large necrotic size and ONFH involving the lateral pillar. The present study further analysed the correlation between the risk value (based on the number of pillars involved and the involvement location) and the collapse rate. The Spearman rank test revealed a significant correlation $(R=1)$, which indicates that $\mathrm{ABC}$ typing is satisfactorily correlated with the collapse risk in ONFH and may be utilized for prediction of collapse. This result was similar to results based on JIC typing (19) and CJFH typing (30). However, the demarcation method for the three pillars according to the $\mathrm{ABC}$ method proposed in the present study was based on biomechanical theory, and this newly proposed typing method considers the anatomical characteristics of the normal femoral head and the stress distribution characteristics of the bone trabeculae inside the femoral head; thus, the demarcation criteria for this typing method are more specific, compared with JIC and CJFH typing. Furthermore, the $\mathrm{ABC}$ typing method utilizes the plane with the maximum necrotic area for typing, which is different from JIC and $\mathrm{CJFH}$, and can better reflect the actual ONFH status, thereby increasing the accuracy of collapse prediction.

Of note, the present study has certain limitations. First, it was a cross-sectional study, so the accuracy of collapse prediction remains to be validated by prospective studies. Furthermore, the ABC typing described in the present study only considered the necrotic area and the necrotic location, and the factor of bone remodelling ability after ONFH was excluded. This exclusion was primarily due to the complexity of the typing issues and for convenience in clinical practice. In any case, the combination of these results with those of a previous study by our group on the sclerotic rim (13) may further increase the accuracy of collapse prediction in ONFH. Finally, the sample size of the present study was small. To further validate the accuracy of the ABC typing method in predicting the collapse of the osteonecrosis-affected femoral, multicentric prospective studies with larger sample sizes should be performed in the future.

In conclusion, the ABC typing method applied in the present study took the lesion size and location into account, and a satisfactory correlation with the collapse risk in $\mathrm{ONFH}$ was determined. The ABC method is easy to perform with reproducibility. The significance of the proposal of this method lies in that the typing outcomes help determine appropriate treatment protocols according to the specific risk of collapse and goals of treatment. In the $\mathrm{ABC}$ typing system, types $\mathrm{A}-\mathrm{C}$ and $\mathrm{AB}$ had a poor prognosis, whereas types $\mathrm{B}$ and $\mathrm{C}$ had a good prognosis, followed by types $\mathrm{BC}$ and A, which suggests that the occurrence of collapse is associated with the necrotic area: The larger the necrotic size, the higher the risk of collapse. Furthermore, collapse occurrence is associated with the necrotic location: The closer to the lateral side of the femoral head, the higher the risk of collapse. The co-existence of a large necrotic area and a lateral necrotic location increases the collapse risk in ONFH. Based on the type classification outcomes, the following is suggested: i) For type $\mathrm{C} \mathrm{ONFH,} \mathrm{special}$ treatment is normally unnecessary as collapse is generally unlikely to occur in this type, and regular follow-ups are enough for patients with this condition. ii) Type B has a low risk of collapse, so that for patients with this type of ONFH, biological treatment (e.g., stem cell transplantation, circulation improvement, medication for bone resorption inhibition and bone formation promotion) may be selected as the major therapeutic method in clinical practice, whereas lifestyle intervention measures are not required. iii) The A and BC types have a considerable risk of collapse, so for patients with these types of ONFH, lifestyle intervention measures and non-supportive cancellous bone transplantation, such as those for weight-bearing protection, reduction of joint load and bone grafting impaction, are necessary in addition to biological treatment. iv) The A-C and AB types have a high risk of collapse, and for affected patients, appropriate therapies for improving biomechanical support (e.g., osteotomy and grafting of the fibula) are required to prevent the occurrence of collapse, apart from biological treatment and lifestyle intervention measures.

\section{Acknowledgements}

The authors would like to thank Prof. Fulei Chu from the Department of Mechanical Engineering (Tsinghua University, Beijing, China) for assistance in using Auto CAD software.

\section{Funding}

No funding was received.

\section{Availability of data and materials}

The analyzed data sets generated during the present study are available from the corresponding author on reasonable request.

\section{Authors' contributions}

$\mathrm{ZZ}$ and TY devised the study plan and led the writing of the article. YuL, XK, and YaL conducted the experiment and collected the data. SH, HD, and YB conducted the analysis. LX supervised the whole process, and gave constructive advice and final approval for the version to be published. The final version of the manuscript has been read and approved by all authors, and each author believes that the manuscript represents honest work.

\section{Ethical approval and consent to participate}

The present study was performed in accordance with the Declaration of Helsinki. Approval was obtained from the Ethics Committee of Guanganmen Hospital of the China Academy of Chinese Medicine Sciences (Beijing, China). Written informed consent was obtained from all participants.

\section{Patient consent for publication}

The patients provided written informed consent for the publication of any associated data and accompanying images.

\section{Competing interests}

The authors declare that they have no competing interests. 


\section{References}

1. Zhao DW and Hu YC: Chinese experts' consensus on the diagnosis and treatment of osteonecrosis of the femoral head in adults. Orthop Surg 4: 125-130, 2012.

2. Microsurgery Department of the Orthopedics Branch of the Chinese Medical Doctor Association; Group from the Osteonecrosis and Bone Defect Branch of the Chinese Association of Reparative and Reconstructive Surgery and Microsurgery and Reconstructive Surgery Group of the Orthopedics Branch of the Chinese Medical Association: Chinese Guideline for the diagnosis and treatment of osteonecrosis of the femoral head in adults. Orthop Surg 9: 3-12, 2017.

3. Wang F and Zhang YH: Advances in research on the cause and pathological mechanisms of non-traumatic ischemic necrosis of femoral head. J Pract Diagnosis Ther 2: 121-123, 2007 (In Chinese).

4. Li ZR: Scientific diagnosis and treatment of osteonecrosis of the femoral head. Chinese J Reparative Reconstructive Surg 9 685-686, 2005

5. Floerkemeier T, Thorey F, Daentzer D, Lerch M, Klages P, Windhagen $\mathrm{H}$ and von Lewinski $\mathrm{G}$ : Clinical and radiological outcome of the treatment of osteonecrosis of the femoral head using the osteonecrosis intervention implant. Int Orthop 35: 489-495, 2011

6. Yu T, Zhang Z, Xie L, Ke X and Liu Y: The influence of traditional Chinese medicine constitutions on the potential repair capacity after osteonecrosis of the femoral head. Complement Ther Med 29: 89-93, 2016.

7. Moya-Angeler J, Gianakos AL, Villa JC, Ni A and Lane JM: Current concepts on osteonecrosis of the femoral head. World J Orthop 6: 590-601, 2015.

8. Mont MA, Cherian JJ, Sierra RJ, Jones LC and Lieberman JR: Nontraumatic osteonecrosis of the femoral head: Where do we stand today? A Ten-year update. J Bone Joint Surg Am 97: 1604-1627, 2015

9. Pierce TP, Elmallah RK, Jauregui JJ, Verna DF and Mont MA: Outcomes of total hip arthroplasty in patients with osteonecrosis of the femoral head-a current review. Curr Rev Musculoskelet Med 8: 246-251, 2015.

10. Rajpura A, Wright AC and Board TN: Medical management of osteonecrosis of the hip: A review. Hip Int 21: 385-392, 2011.

11. Gillet C, Dalla Valle A, Gaspard N, Spruyt D, Vertongen P, Lechanteur J, Rigutto S, Dragan ER, Heuschling A, Gangji V and Rasschaert J: Osteonecrosis of the Femoral head: Lipotoxicity exacerbation in MSC and modifications of the bone marrow fluid. Endocrinology 158: 490-502, 2017.

12. Fukushima W, Fujioka M, Kubo T, Tamakoshi A, Nagai M and Hirota Y: Nationwide epidemiologic survey of idiopathic osteonecrosis of the femoral head. Clin Orthop Relat Res 468 : 2715-2724, 2010

13. Yu T, Xie L, Zhang Z, Ke X and Liu Y: Prediction of osteonecrosis collapse of the femoral head based on the proportion of the proximal sclerotic rim. Int Orthop 39: 1045-1050, 2015.

14. Sugano N, Takaoka K, Ohzono K, Matsui M, Masuhara K and Ono K: Prognostication of nontraumatic avascular necrosis of the femoral head. Significance of location and size of the necrotic lesion. Clin Orthop Relat Res 303: 155-164, 1994.

15. Morita D, Hasegawa Y, Okura T, Osawa Y and Ishiguro N Long-term outcomes of transtrochanteric rotational osteotomy for non-traumatic osteonecrosis of the femoral head. Bone Joint J 99-B: 175-183, 2017.

16. Koo KH and Kim R: Quantifying the extent of osteonecrosis of the femoral head. A new method using MRI. J Bone Joint Surg Br 77: 875-880, 1995 .
17. Nishii T, Sugano N, Ohzono K, Sakai T, Sato Y and Yoshikawa H: Significance of lesion size and location in the prediction of collapse of osteonecrosis of the femoral head: A new three-dimensional quantification using magnetic resonance imaging. J Orthop Res 20: 130-136, 2002.

18. Kerboul M, Thomine J, Postel M and Merle DR: The conservative surgical treatment of idiopathic aseptic necrosis of the femoral head. J Bone Joint Surg Br 56: 291-296, 1974.

19. Min BW, Song KS, Cho CH, Lee SM and Lee KJ: Untreated asymptomatic hips in patients with osteonecrosis of the femoral head. Clin Orthop Relat Res 466: 1087-1092, 2008.

20. Hernigou P and Lambotte JC: Volumetric analysis of osteonecrosis of the femur. Anatomical correlation using MRI. J Bone Joint Surg Br 83: 672-675, 2001.

21. Gardeniers JWM: Report of the Committee of Staging and Nomenclature. ARCO News Letter 5: no. 2: 79-82, 1993.

22. Steinberg ME, Hayken GD and Steinberg DR: A quantitative system for staging avascular necrosis. J Bone Joint Surg 77: 34-41, 1995.

23. Li ZR, Liu ZH, Sun W, Shi ZC, Wang BL and Zhao FC: The classification of osteonecrosis of the femoral head based on the three pillars structure: China-Japan Friendship Hospital (CJFH) classification. Chin J Orthop 6: 515-520, 2012 (In Chinese).

24. Li ZR: Osteonecrosis. The People's Health Publishing House, Beijing, 2012.

25. Ahn AC and Grodzinsky AJ: Relevance of collagen piezoelectricity to 'Wolff's Law': A critical review. Med Eng Phys 31: 733-741, 2009

26. Tsubota K, Suzuki Y, Yamada T, Hojo M, Makinouchi A and Adachi T: Computer simulation of trabecular remodeling in human proximal femur using large-scale voxel FE models: Approach to understanding Wolff's law. J Biomech 42: 1088-1094, 2009.

27. Chen JH, Liu C and You L: Boning up on Wolff's Law: Mechanical regulation of the cells that make and maintain bone. J Biomech 43: 108-118, 2010.

28. Ma XL, Li HT and Ma JX: Biomechanical properties of principle compressive trabecular bone in proximal femur. Biome Eng Clin Med 2: 118-122, 2012 (In Chinese).

29. Zhou GQ, Pang ZH, Chen QQ, He W, Chen ZQ, Chen LL and Li ZQ: Reconstruction of the biomechanical transfer path of femoral head necrosis: A subject-specific finite element investigation. Comput Biol Med 52: 96-101, 2014.

30. Li Z, Sun W, Gao F, Liu Z, Shi Z and Wang B: Comparative evaluation of osteonecrosis of the femoral head classification: CJFH classification versus JIC classification. J Hip Surg 1: 44-49, 2017.

31. Herring JA, Neustadt JB, Williams JJ, Early JS and Browne RH: The lateral pillar classification of Legg-Calvé-Perthes disease. J Pediatr Orthop 12: 143-150, 1992.

32. Zhang ZN: 3S system-based typing for osteonecrosis and its relation with Chinese medicine-based constitution. Dissertation China Acad Chin Med Sci, 2015 (In Chinese).

33. Zhang ZN, Xie LM and Yu T: Comparative study on the differentiation of osteonecrosis of femoral head at different sections based on three pillars structure. Chin J Tradit Med Traumatol Orthop 25: 28-31, 2017 (In Chinese).

34. Yu T, Xie L and Chu F: A sclerotic rim provides mechanical support for the femoral head in osteonecrosis. Orthopedics 38: e374-e379, 2015

35. Kim YM, Ahn JH, Kang HS and Kim HJ: Estimation of the extent of osteonecrosis of the femoral head using MRI. J Bone Joint Surg Br 80: 954-958, 1998. 